\title{
Underwater versus conventional endoscopic mucosal resection for colorectal lesions: a systematic review and meta-analysis
}

\section{(ㄷ)(우우}

Authors

Rajat Garg1', Amandeep Singh², Babu P. Mohan³, Gautam Mankaney², Miguel Regueiro², Prabhleen Chahal ${ }^{2}$

Institutions

1 Department of Hospital Medicine, Cleveland Clinic, Ohio, United States

2 Department of Gastroenterology, Hepatology and Nutrition, Digestive Diseases and Surgery Institute; Cleveland Clinic, Cleveland, Ohio, United States

3 Department of Inpatient Medicine, University of Arizona, Banner University Medical Center, Tucson, Arizona, United States

submitted 24.6.2020

accepted after revision 21.9.2020

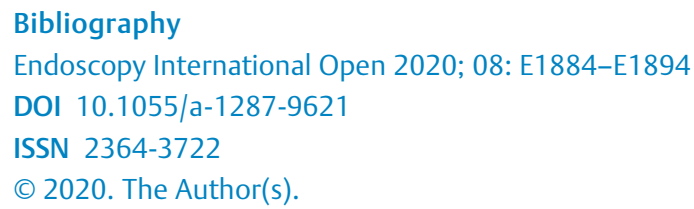
Commons Attribution-NonDerivative-NonCommercial License, permitting copying and reproduction so long as the original work is given appropriate credit. Contents may not be used for commecial purposes, or adapted, remixed, transformed or built upon. (https://creativecommons.org/licenses/by-nc-nd/4.0/)

\section{Corresponding author}

Rajat Garg, MD, Cleveland Clinic Foundation - Internal Medicine, 9500 Euclid Avenue, Cleveland Ohio 44195, United States

Fax: +1-4403128588

drgargrajat@gmail.com

Supplementary material is available under https://doi.org/10.1055/a-1287-9621

\section{ABSTRACT}

Background and study aims Underwater endoscopic mucosal resection (UEMR) for colorectal polyps has been reported to have good outcomes in recent studies. We conducted a systematic review and meta-analysis comparing the effectiveness and safety of UEMR to conventional EMR (CEMR).

Methods A comprehensive search of multiple databases (through May 2020) was performed to identify studies that reported outcome of UEMR and CEMR for colorectal lesions. Outcomes assessed included incomplete resection, rate of recurrence, en bloc resection, adverse events (AEs) for UEMR and CEMR.

Results A total of 1,651 patients with 1,704 polyps were included from nine studies. There was a significantly lower rate of incomplete resection (odds ratio [OR]: 0.19 (95\% confidence interval $(\mathrm{Cl}), 0.05-0.78, P=0.02)$ and polyp recurrence (OR: $0.41,95 \% \mathrm{Cl}, 0.24-0.72, P=0.002$ ) after UEMR. Compared to CEMR, rates overall complications (relative risk [RR]: $0.66(95 \% \mathrm{Cl}, 0.48-0.90)(P=0.008)$, and intra-procedural bleeding (RR: $0.59,95 \% \mathrm{Cl}, 0.41-$ $0.84, P=0.004$ ) were significantly lower with UEMR. The recurrence rate was also lower for large non-pedunculated polyps $\geq 10 \mathrm{~mm}$ (OR $0.24,95 \% \mathrm{Cl}, 0.10-0.57, P=0.001)$ and $\geq 20 \mathrm{~mm}$ (OR $0.14,95 \% \mathrm{Cl}, 0.02-0.72, P=0.01$ ). The rates of en bloc resection, delayed bleeding, perforation and post-polypectomy syndrome were similar in both groups $(P>0.05)$.

Conclusions In this systematic review and meta-analysis, we found that UEMR is more effective and safer than CEMR with lower rates of recurrence and AEs. UEMR use should be encouraged over CEMR.

\section{Introduction}

Endoscopic mucosal resection (EMR) is the primary treatment modality for large and/or sessile colorectal polyps [1,2]. In the conventional EMR (CEMR) technique, a submucosal fluid injection creates a cushion to separate the deeper muscularis mucosa from the superficial epithelial layer that contains the lesion.
The submucosal injection is performed to prevent full thickness perforation and deep thermal injury by increasing the distance between the electrocautery current and the transmural space [3]. Submucosal injection assisted EMR has been widely accepted technique for colorectal lesions and has largely replaced surgical resection. The European society of gastrointestinal endoscopy (ESGE) recommends EMR with submucosal injection 
for sessile or flat polyps $\geq 10 \mathrm{~mm}$ in size [3]. The alternative approaches, including surgery and endoscopic submucosal dissection (ESD), are costly, more time consuming, require more resources, and ESD is not readily available in the US [4,5]. In spite of these advantages, CEMR for large polyps have been associated with high rates of incomplete resection and local recurrence of $15 \%$ to $30 \%$ on follow up [2, 5-7].

In underwater EMR (UEMR), the mucosa and submucosa float away from muscularis propria, facilitating polyectomy [8]. Removal of intraluminal air also decreases colonic wall tension, which permits the colon wall to assume its natural collapsed state. It was first described by Binmoeller and colleagues in 2012 based on observation during endosonography [8]. Multiple studies have reported good results of UEMR along with low rate of adverse events [9-13], but data-comparing UEMR to CEMR has not been systematically reviewed. We performed a systematic review and meta-analysis comparing the safety and effectiveness of UEMR and CEMR for the resection of colorectal polyps.

\section{Methods}

\section{Search strategy}

We conducted a comprehensive search of several databases from inception to May 2020. The databases included Ovid MEDLINE and Epub Ahead of Print, In-Process and other non-indexed citations, Ovid Embase, Ovid Cochrane Central Register of Controlled trials, Ovid Cochrane Database of Systematic Reviews, and Scopus. An experienced medical librarian using inputs from the study authors helped with the literature search. Controlled vocabulary supplemented with keywords was used to search for studies of interest. The full search strategy is available in Appendix 1. The MOOSE and PRISMA checklist were followed and are provided in Appendix 2 and Appendix 3 [14, 15].

\section{Study selection}

All studies that reported clinical outcomes of CEMR and UEMR were included, irrespective of sample-size, inpatient/ outpatient setting, and geography, as long as they provided any data needed for the analysis.

Studies done in pediatric population (Age $<18$ years), and studies not published in English language were excluded. In cases of multiple publications from the same cohort and/or overlapping cohorts, data from the most recent and/or most appropriate comprehensive report were retained.

\section{Data abstraction and quality assessment}

Data on study-related outcomes in the individual studies were abstracted onto a standardized form and quality score independently by two authors (RG and BPM). Primary study authors were contacted via email for additional data or any clarification on data.

The Newcastle-Ottawa scale for cohort studies and Jadad score for randomized control trials was used to assess the quality of studies [16]. This Newcastle Ottawa quality score consists of eight questions and Jadad score consists of five questions, the details of which are provided in Supplementary Table 1.

\section{Outcomes assessed}

The primary outcome was rate of any incomplete resection. Incomplete resection was defined as presence of macroscopic residual polyp based on endoscopist assessment reported by study authors. Secondary outcomes included R0 resection, recurrence/residual polyp on follow up colonoscopy, adverse events and en bloc resection. The $\mathrm{R} 0$ resection was defined as margins clear of any abnormal tissue based on histologic assessment. The recurrence/residual rate was based on first follow up colonoscopy and presence of abnormal lesion at the site of previous intervention. The recurrence/residual polyp was described on endoscopic and histologic assessment by study authors. The adverse events were further classified into intra-procedural or delayed bleeding, perforation and post-polypectomy syndrome (PPS). Intra-procedural bleeding was defined as immediate bleeding requiring endoscopic hemostasis and delayed bleeding was defined as post-procedural bleeding within 2 to 4 weeks of intervention. En bloc resection was defined as lesion resection as one piece rather than in multiple small pieces

Subgroup analysis was performed for non-pedunculated polyps $\geq 10 \mathrm{~mm}$ and $\geq 20 \mathrm{~mm}$.

\section{Statistical analysis}

We used meta-analysis techniques to calculate the odds ratio for resection outcomes and relative risks for complications outcome using inverse variance equation and random-effects model as described by DerSimonian and Laird [17]. We assessed heterogeneity between study-specific estimates by using Cochran Q statistical test for heterogeneity, [18-20] and the $I^{2}$ statistics $[21,22]$. In this, values of $<30 \%, 30 \%$ to $60 \%, 61 \%$ to $75 \%$, and $>75 \%$ were suggestive of low, moderate, substantial, and considerable heterogeneity, respectively [23]. If heterogeneity was present, we attempted to assess the reasons of the heterogeneity. $P<0.05$ was used to define statistical significance between the groups.

All analyses were performed using RevMan 5.0 (Cochrane collaboration) statistical software [24].

\section{Results}

\section{Search results and population characteristics}

From an initial 242 studies, 144 records were screened and 41 full-length articles were assessed. Nine studies were included in the final analysis that reported and compared outcomes of UEMR and CEMR [13, 25-32]. The schematic diagram of study selection is illustrated in Supplementary Fig. 1. In one study, we excluded some small polyps $<10 \mathrm{~mm}$ as they were resected with underwater cold snare rather than EMR and did not meet inclusion criteria [32].

A total of 1,651 patients with 1,704 polyps were included from 9 studies in the final analysis. Out of 1,704 polyps, 891 were resected by CEMR and 813 were resected by UEMR. The mean age ranged from 62.3 to 70 years and majority of the pa- 
tients were males $(66.4 \%, n=1,132)$. The mean polyp size ranged from $9.9 \mathrm{~mm}$ to $30.2 \mathrm{~mm}$ in CEMR group and $9.9 \mathrm{~mm}$ to $27.5 \mathrm{~mm}$ in UEMR group. The type of polyp based on Paris classification was available in seven studies including 1,266 (88.7\%) non-pedunculated polyps (647 CEMR and 619 UEMR). The mean duration of procedure ranged from 3.4 to 26.4 minutes in CEMR group and 1.5 to 13.3 minutes in UEMR group. The baseline characteristics and data on assessed outcomes are shown in $>$ Table 1 and $>$ Table 2 , respectively.

There were seven studies reporting outcomes on non-pedunculated polyps $\geq 10 \mathrm{~mm}$. Amongst the total of 1,266 nonpedunculated polyps, 95.1\% $(n=1,204,612$ CEMR and 592 UEMR) were $\geq 10 \mathrm{~mm}$ and $23.2 \%(n=294,166$ CEMR and 128 UEMR) were $\geq 20 \mathrm{~mm}$. Data on assessed outcomes for non-pedunculated polyps $\geq 10 \mathrm{~mm}$ and $\geq 20 \mathrm{~mm}$ are shown in Supplementary Table 2 and Supplementary Table 3 respectively.

\section{Characteristics and quality of included studies}

Five studies were retrospective $[13,25,27,29,31]$ one prospective [28] and three were randomized controlled trials [26,30, 32]. Six studies were full-text articles [13, 25, 28-30,32] and three were published abstracts $[26,27,31]$. Amongst the six cohort studies, four were of high quality and two were medium quality. Based on Jadad score, two of three randomized trials were of good quality and one was of poor quality. The quality assessment is shown in Supplementary Table 1.

\section{Meta-analysis outcomes}

The rate of incomplete resection in UEMR group was significantly lower than CEMR group (odds ratio [OR]: 0.19, $95 \%$ confidence interval $[\mathrm{Cl}], 0.05-0.78, \mathrm{I}^{2}=23, P=0.02$ ) ( $\mathrm{Fig}$. 1a). The rate of $\mathrm{R} 0$ resection was provided in two studies. From these studies, we noticed significantly higher odds of $R 0$ resection in UEMR as compared to CEMR with OR of 2.20 (95\% Cl, 1.26$3.83, I^{2}=0, P=0.0005$ ) ( Fig. 1b). The recurrence rate and follow up was reported in six studies. A total of 338 and 210 patients underwent follow up in CEMR and UEMR group respectively. The follow-up duration ranged from 3 to 14 months after the index procedure. The recurrence rate was significantly lower in patients who underwent UEMR than in patients who underwent CEMR (OR: $0.41,95 \% \mathrm{Cl}, 0.24-0.72, \mathrm{I}^{2}=0, P=0.002$ ) ( Fig. 1c).

There were a total of 160 adverse events, 91 (10.2\%) in CEMR group and 59 (7.2\%) in UEMR group. The most common complication was intra-procedural bleeding $(73.7 \%, n=118,70$ in CEMR group and 38 in UEMR group) followed by delayed bleeding $(21.2 \%, n=34)$, three cases of perforation, and two cases of PPS syndrome. There was one report of transient bacteremia after UEMR [26] and three cases (2 in CEMR and 1 in UEMR group) of muscle layer injury without perforation [13]. The rate of overall complications was significantly lower with UEMR compared to CEMR with relative risk (RR) of 0.66 (95\% $\mathrm{Cl}, 0.48-0.90, \mathrm{I}^{2}=0$ ), $P=0.008$ ( $\mathbf{F i g . 2 a}$ ). This was primarily derived from significantly less incidence of intra-procedural bleeding in UEMR with RR of $0.59\left(95 \% \mathrm{Cl}, 0.41-0.84, \mathrm{I}^{2}=0\right), P$ $=0.004$ ( Fig. $\mathbf{2 b}$ ). There were $16(1.8 \%$ ) cases of delayed bleeding, two cases of perforation and one case of PPS in
CEMR group, compared to 18 (2.2\%) cases of delayed bleeding, one case of perforation and one case of PPS in UEMR group. Overall, the incidence of delayed bleeding (RR: $1.58,95 \% \mathrm{Cl}$, $0.75-3.33, I^{2}=1, P=0.24$ ), perforation (RR: $0.89,95 \% \mathrm{Cl}$, $0.14-5.62, \mathrm{I}^{2}=0, P=0.90$ ), and PPS (RR: $1.08,95 \% \mathrm{Cl}, 0.11-$ $10.27, I^{2}=0, P=0.94$ ) were low in our study population and did not differ significantly amongst both groups ( $>$ Fig. 3a, $>$ Fig. 3b, - Fig. 3c).

There was a higher trend of en bloc resection in UEMR group as compared to CEMR (OR: 1.33, $95 \% \mathrm{Cl}, 0.72-2.44, \mathrm{I}^{2}=82 \%$ ), but this difference did not reach statistical significance $(P=$ $0.36)$. Due to presence of substantial heterogeneity, we further inspected data from only prospective studies. Compared to CEMR, the rates of en bloc resections in UEMR were $\sim 2.5$-fold higher (OR: 2.48 (95\% Cl, 1.57-3.93, $\left.\mathrm{I}^{2}=39, P<0.001\right)$ with less heterogeneity in prospective studies (Supplementary Fig. 2).

\section{Subgroup analysis of non-pedunculated polyps $\geq 10 \mathrm{~mm}$}

There were seven studies that provided data on polyp characteristics. A total of 1,204 non-pedunculated polyps $\geq 10 \mathrm{~mm}$ were included in the analysis. Compared to CEMR, odds of incomplete resection (OR: $0.26,95 \% \mathrm{Cl}, 0.03-2.54, \mathrm{I}^{2}=55, P=$ 0.11 ) were not significant but recurrence rate (OR: $0.24,95 \%$ $\left.\mathrm{Cl}, 0.10-0.57, \mathrm{I}^{2}=0, P=0.001\right)$ was significantly lower in patients who underwent UEMR. There were no significant differences in rates of overall adverse events, delayed bleeding, perforation and PPS in both groups, however, UEMR had significantly lower risk of intra-procedural bleeding (RR: 0.64, $95 \%$ $\left.\mathrm{Cl}, 0.44-0.94, \mathrm{I}^{2}=0, P=0.02\right)$. The UEMR has again higher trend of en bloc resection with OR of $1.44\left(95 \% \mathrm{Cl}, 0.74-2.78, \mathrm{I}^{2}=84\right.$, $P=0.28)$ as compared to CEMR. This effect was stronger on including only prospective studies (OR: $2.48,95 \% \mathrm{Cl}, 1.57-3.93$, $\left.\mathrm{I}^{2}=39, P<0.001\right)$. Two studies did not show any statistically significant difference for R0 resection in UEMR vs. CEMR groups (OR: $1.62,95 \% \mathrm{Cl}, 0.86-3.04, \mathrm{I}^{2}=52, P=0.15$ ). These results are summarized in $>$ Table 3 .

\section{Subgroup analysis of non-pedunculated polyps $\geq 20 \mathrm{~mm}$}

There were five studies that provided data on outcomes of nonpedunculated polyps of size $\geq 20 \mathrm{~mm}$. Compared to CEMR, there was no significant difference in rate of incomplete resection (OR: $0.86,95 \% \mathrm{Cl}, 0.08-8.79, \mathrm{I}^{2}=11 \%, P=0.29$ ) and en bloc resection (OR: $0.90,95 \% \mathrm{Cl}, 0.38-2.17, \mathrm{I}^{2}=58 \%, \mathrm{p}=0.82$ ) with UEMR. There was again significantly low rate of recurrence with $\mathrm{OR}$ of $0.14\left(95 \% \mathrm{Cl}, 0.02-0.72, \mathrm{I}^{2}=11 \%, P=0.01\right)$ after UEMR as compared to CEMR. There was no difference in rates of complication in both groups with overall complications (RR: 2.17, $\left.95 \% \mathrm{Cl}, 0.77-6.17, \mathrm{I}^{2}=0, P=0.15\right)$, intra-procedural bleeding (RR: $0.85,95 \% \mathrm{Cl}, 0.15-4.73, \mathrm{I}^{2}=74 \%, P=0.85$ ), delayed bleeding (RR: $1.77,95 \% \mathrm{Cl}, 0.23-13.34, \mathrm{I}^{2}=0 \%, \mathrm{p}=$ 0.84 ), perforation (RR: $0.89,95 \% \mathrm{Cl}, 0.09-8.39, \mathrm{I}^{2}=0, P=$ 0.92 ), and PPS (RR: 0.92 (95\% Cl, 0.13-6.41, $I^{2}=0, P=0.93$ ). We were unable to compare rate of $\mathrm{R} 0$ resection due to only 


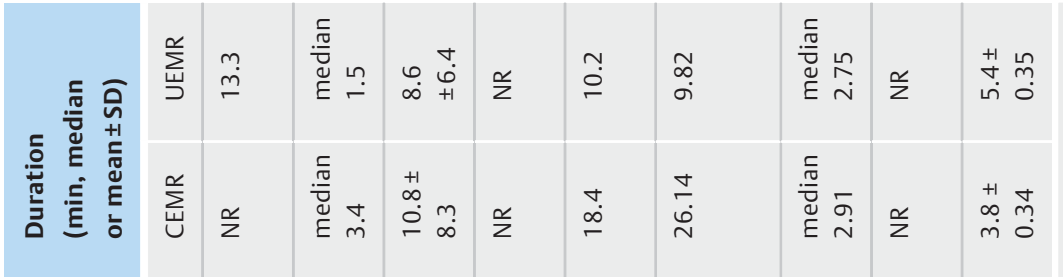

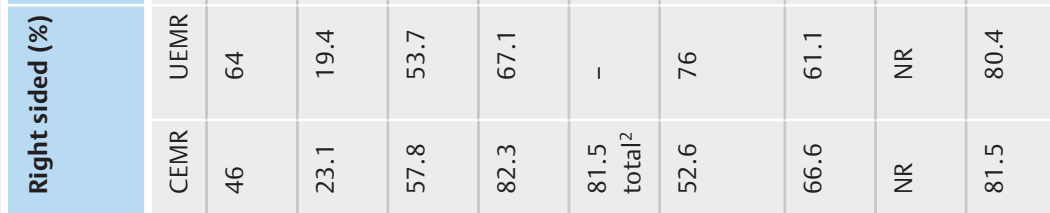

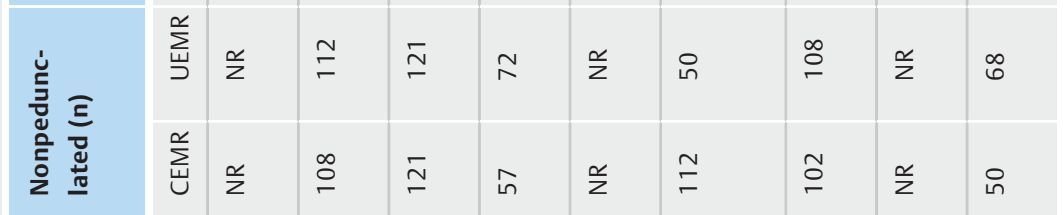

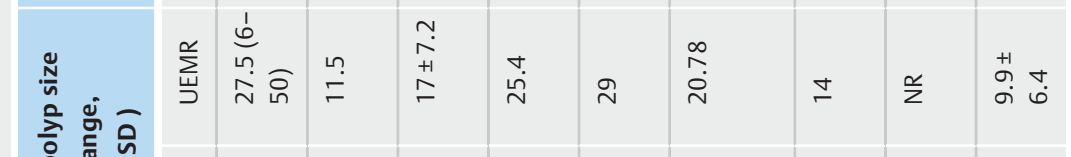

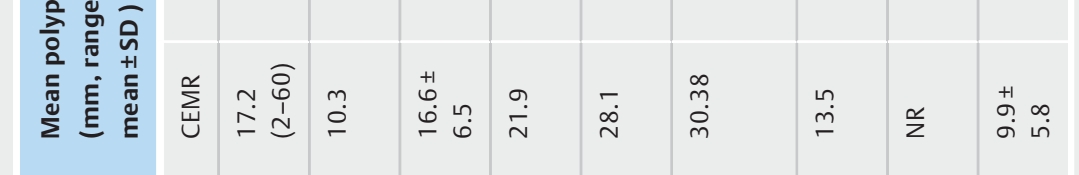

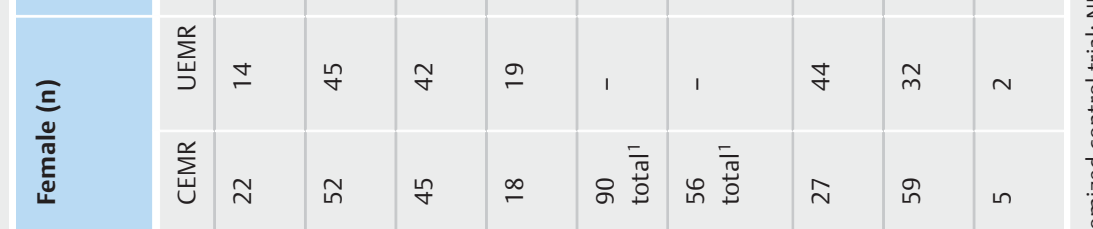

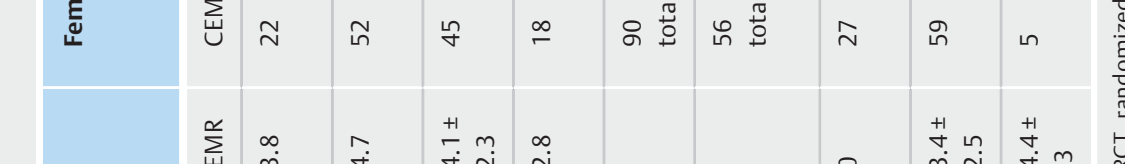

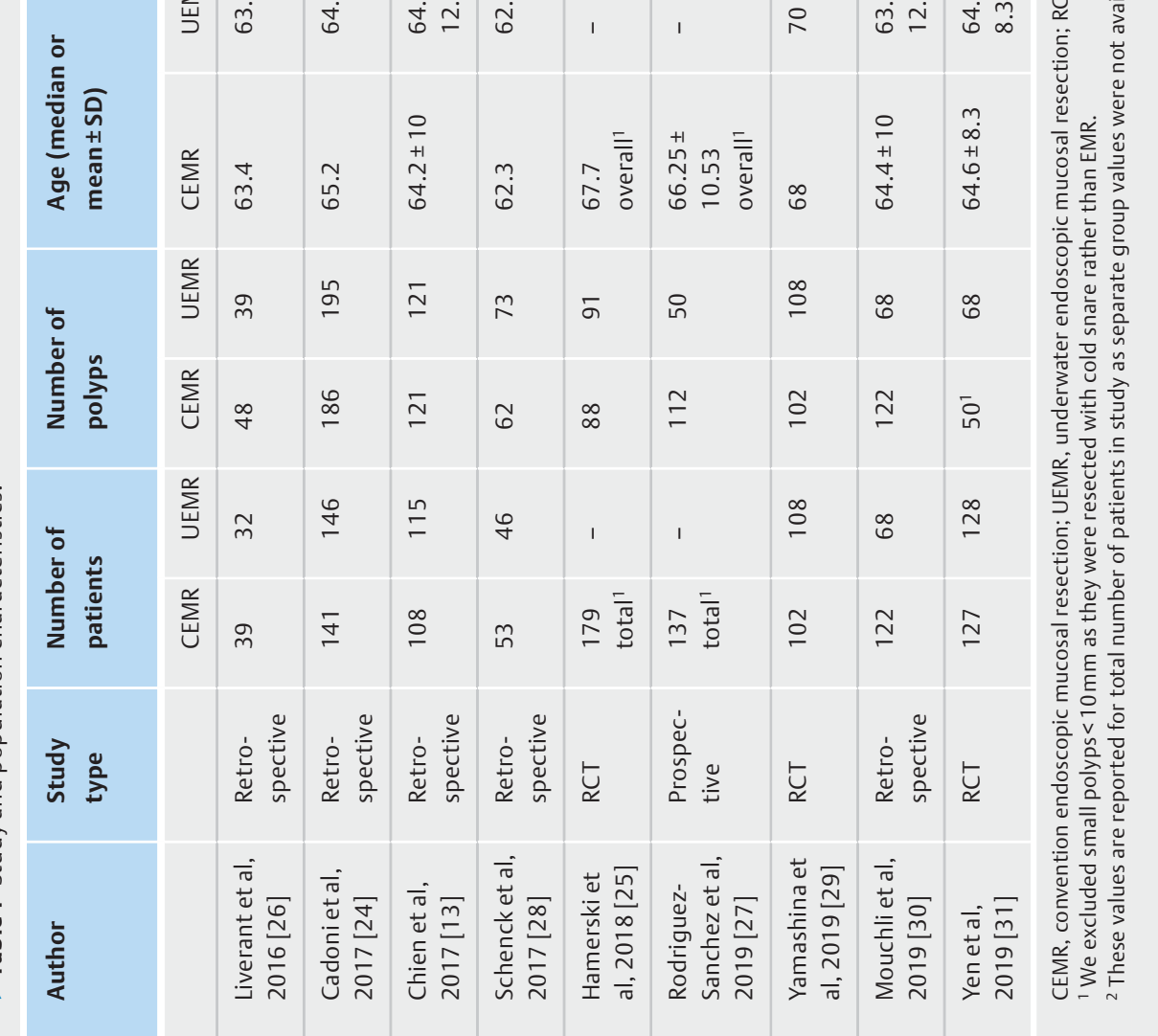




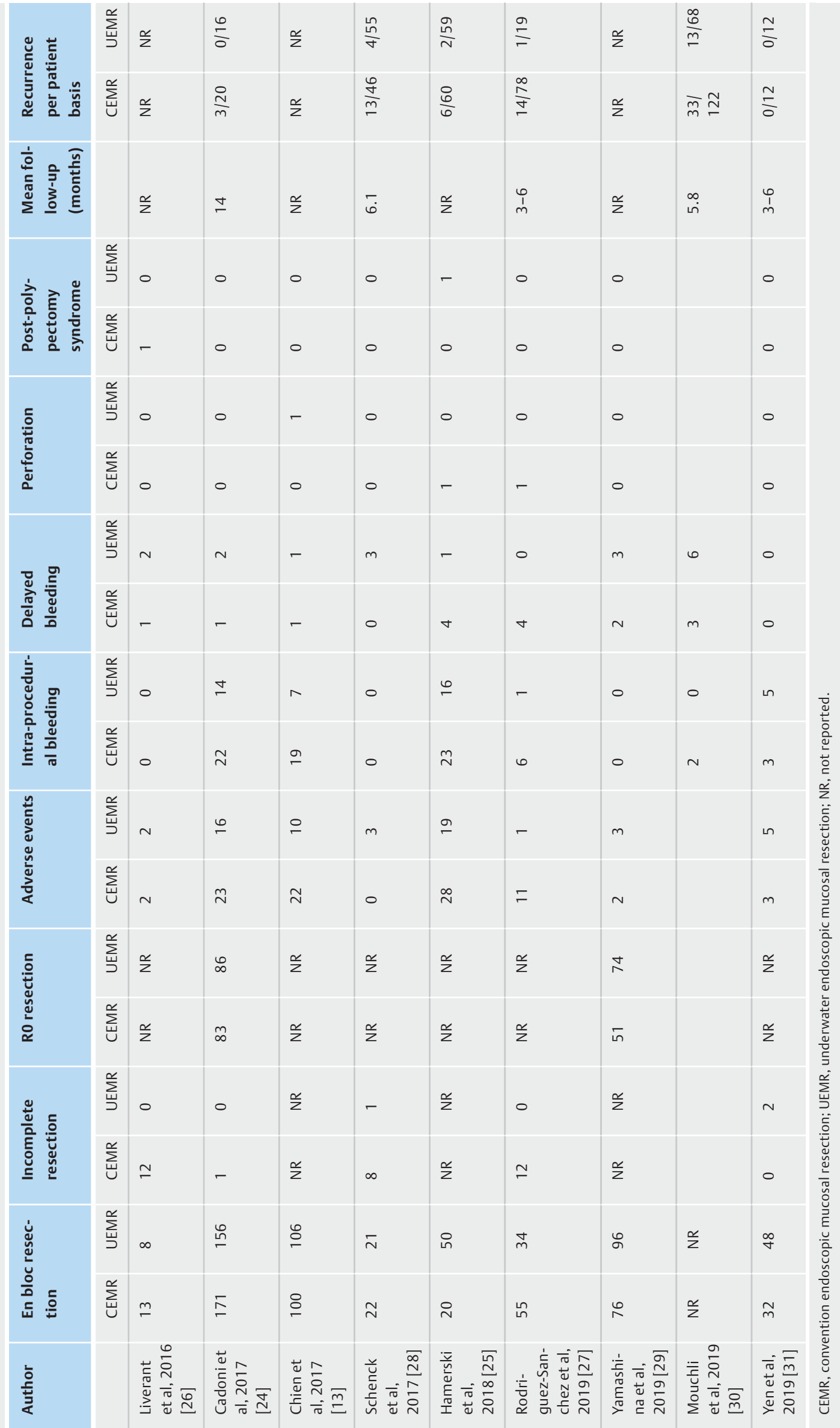




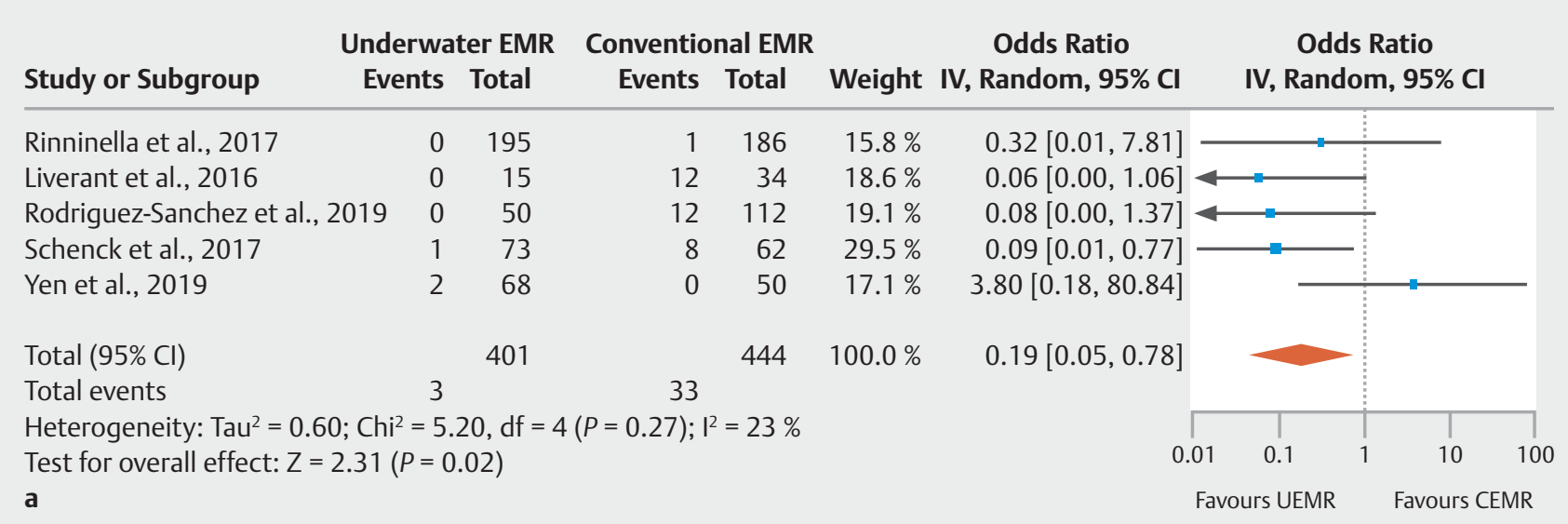

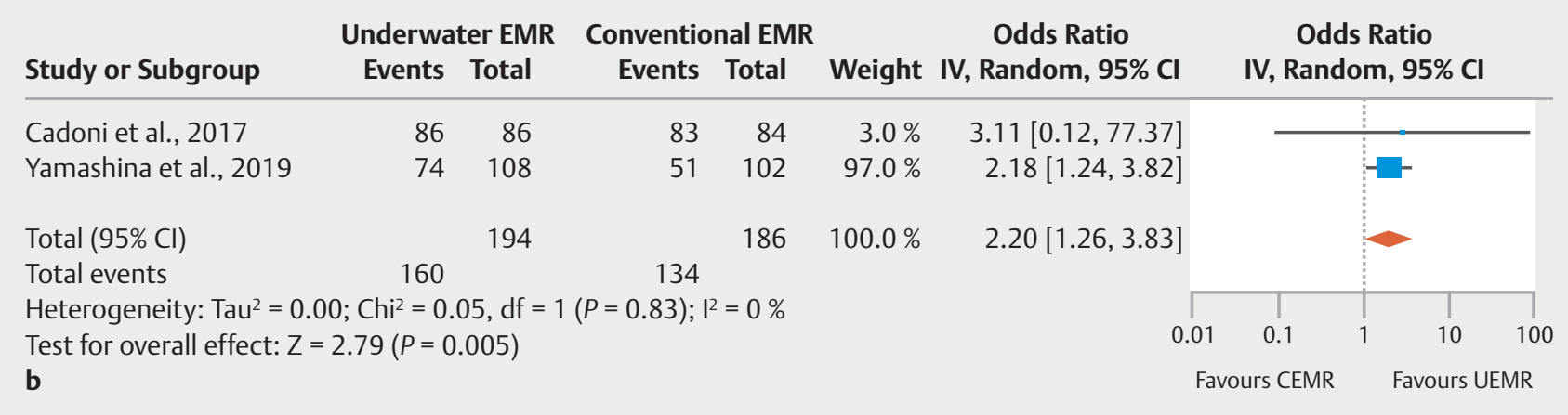

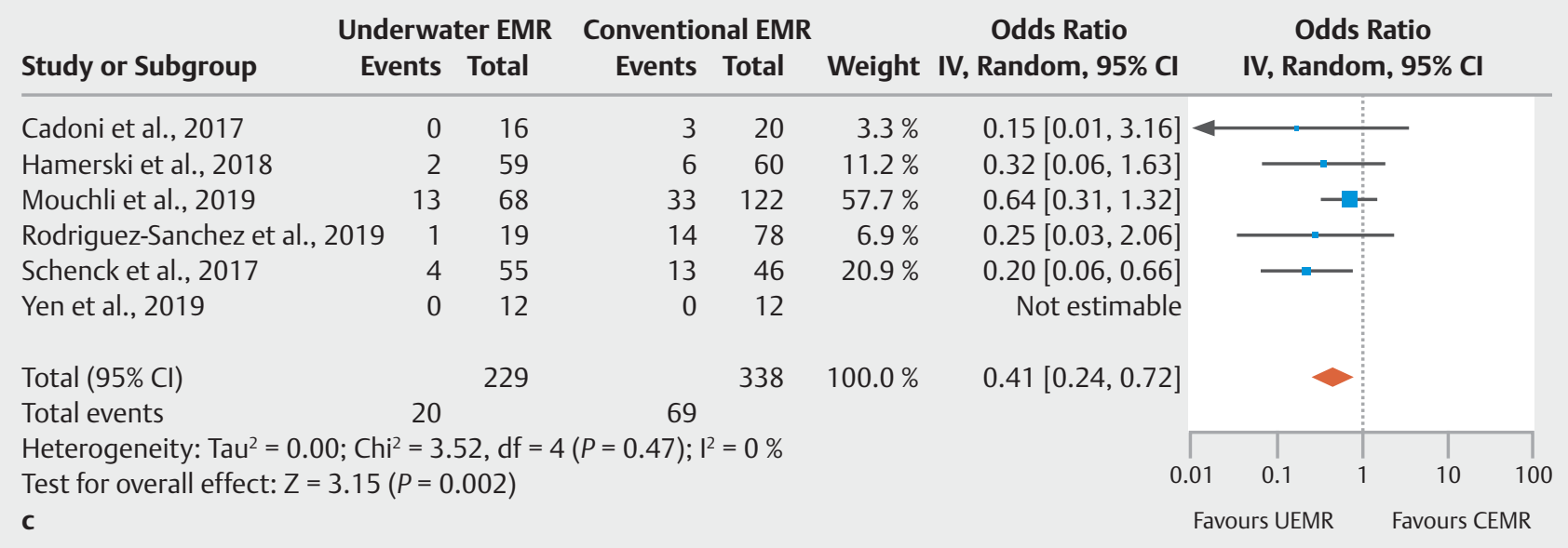

Fig. 1 Forest plot showing odds ratio of a incomplete resection, $\mathbf{b}$ R0 resection, and $\mathbf{c}$ recurrence comparing conventional and underwater endoscopic mucosal resection.

one study reporting this outcome for polyps $>20 \mathrm{~mm}$. These results are also summarized in $>$ Table 3.

\section{Validation of meta-analysis results}

\section{Sensitivity analysis}

To assess whether any one study had a dominant effect on the meta-analysis, we excluded one study at a time and analyzed its effect on the main summary estimate. On this analysis, no single study significantly affected the outcome or the heterogeneity.

\section{Heterogeneity}

We assessed dispersion of the calculated rates using $\mathrm{I}^{2}$ percentage values. The $\mathrm{I}^{2}$ tell us what proportion of the dispersion is true vs. chance [20]. The $\mathrm{I}^{2}$ is reported along with results in - Table 3. Overall, there was low heterogeneity in our study outcomes

\section{Publication bias}

Publication bias was not assessed due to less than ten studies being included in the meta-analysis. 


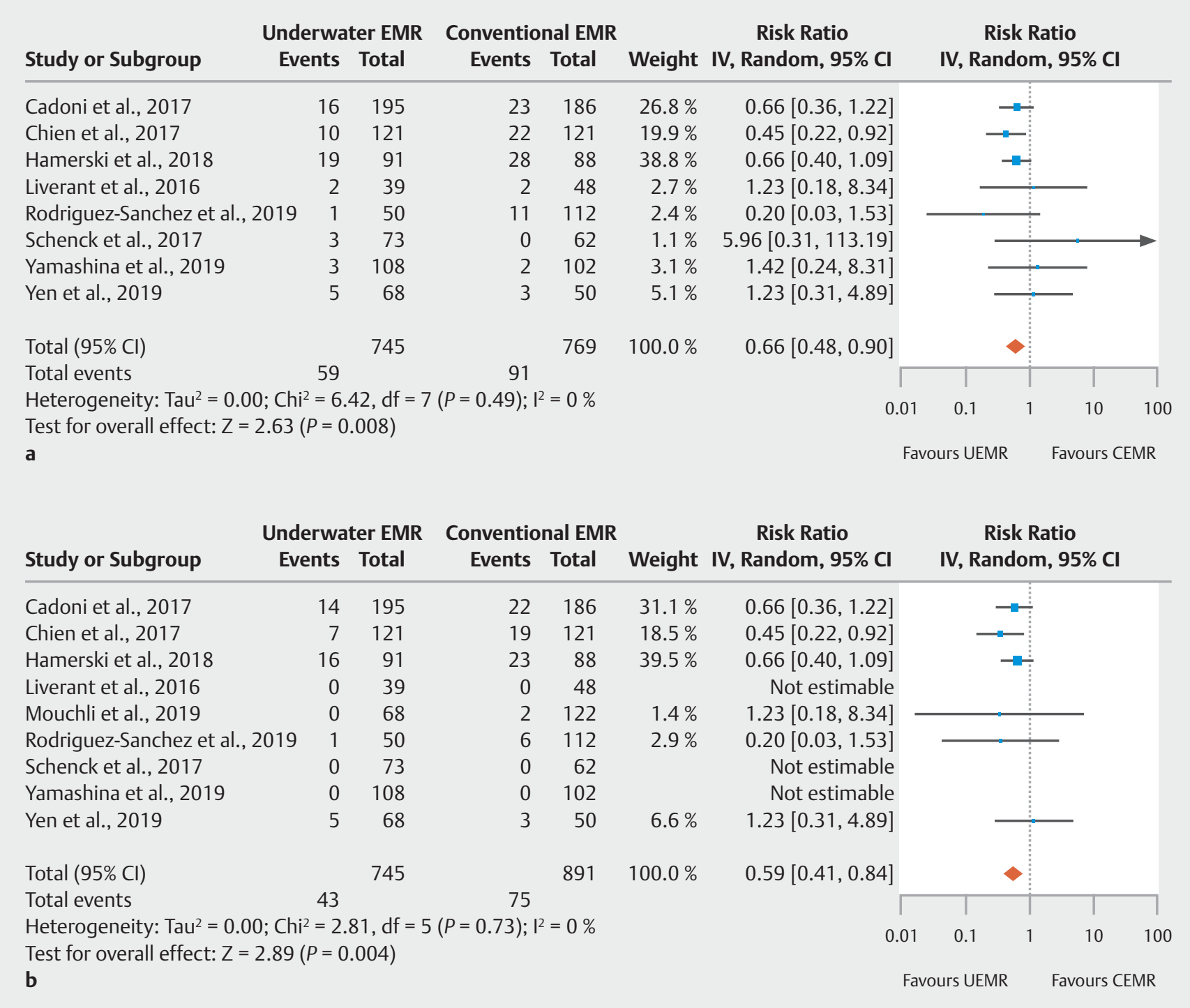

Fig. 2 Forest plot showing relative risk of $\mathbf{a}$ adverse events and $\mathbf{b}$ intra-procedural bleeding comparing conventional and underwater endoscopic mucosal resection.

\section{Discussion}

Our analysis demonstrates that UEMR was significantly associated with less rates of incomplete resection (OR: $0.19,95 \%$ $\mathrm{Cl}, 0.05-0.78, P=0.02$ ) and recurrence (OR: $0.41,95 \% \mathrm{Cl}$, $0.24-0.72, P=0.002)$ of colorectal polyps as compared to CEMR. In addition, UEMR was associated with almost half the risk of complications as compared to CEMR. This was mostly evident by significantly lesser odds of intra-procedural bleeding whereas odds of delayed bleeding, perforation and PPS were similar in both groups. UEMR has double the rate of $\mathrm{R} 0$ resection and is almost three times likely to lead to en bloc resection as compared to CEMR. UEMR advantages were also significantly demonstrated for non-pedunculated polyps $\geq 10 \mathrm{~mm}$ in terms of intra-procedural bleeding and recurrence rate. Even in nonpedunculated polyps $\geq 20 \mathrm{~mm}$, recurrence rates were significantly lower in patients undergoing UEMR. Our study is the lar- gest and first meta-analysis reporting and comparing outcomes of UEMR to CEMR.

Although UEMR has many advantages over CEMR, the major advantages are higher rates of complete resection and en bloc resection with significantly lower rates of recurrence. These effects were also evident in non-pedunculated polyps $>10 \mathrm{~mm}$ in size. UEMR was more effective in resection of larger lesion compared to CEMR. We speculate the advantage of complete resection likely translates into low rate of recurrence on follow up endoscopy. In addition, piecemeal resection has been reported to be an independent significant factor for local recurrence after CEMR [33]. This is extremely significant finding especially in real world setting where patient compliance and behavior plays a major role in follow up. Moreover, lower rates of recurrence will likely translate into lower cost and overall decreased burden on healthcare resources when applied to large population. 


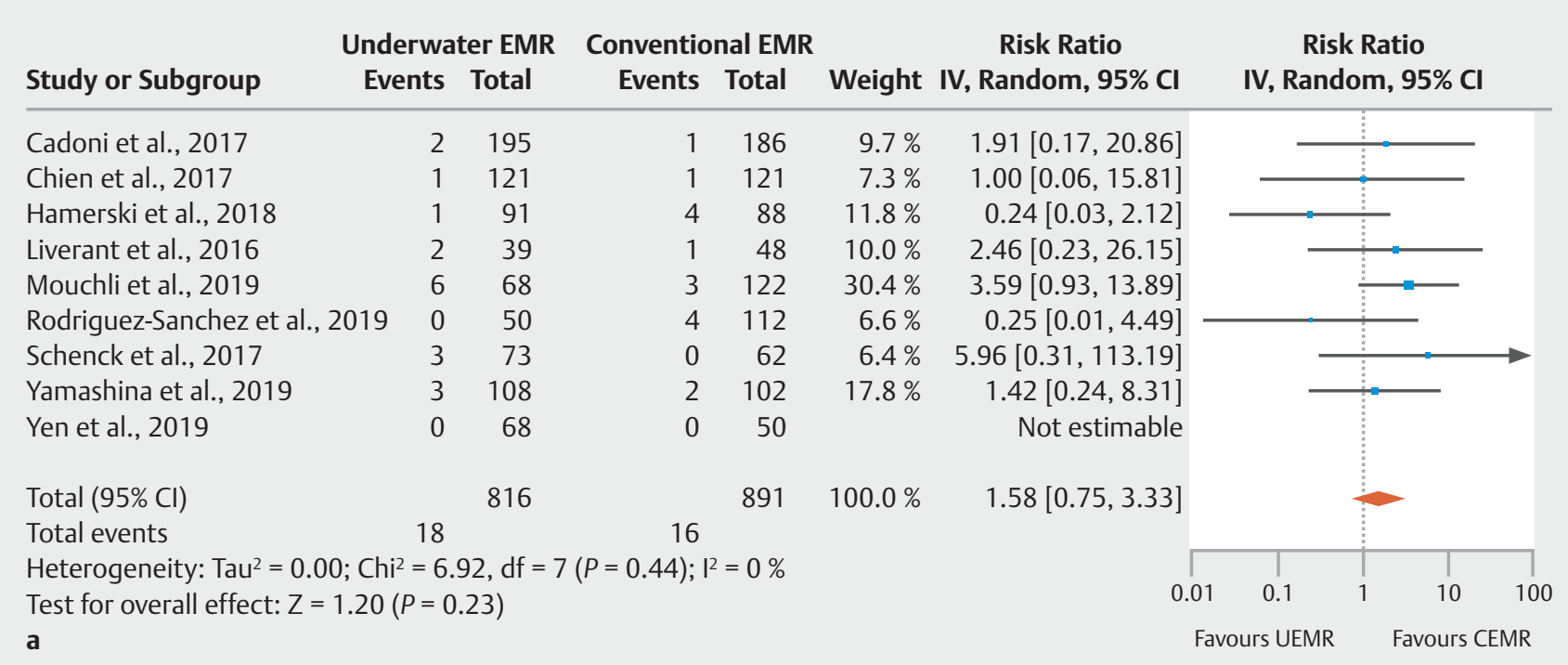

\begin{tabular}{|c|c|c|c|c|}
\hline \multirow[b]{2}{*}{ Study or Subgroup } & \multicolumn{2}{|c|}{ Underwater EMR } & \multicolumn{2}{|c|}{ Conventional EMR } \\
\hline & & Total & Events & Total \\
\hline Cadoni et al., 2017 & 0 & 195 & 0 & 186 \\
\hline Chien et al., 2017 & 1 & 121 & 0 & 121 \\
\hline Hamerski et al., 2018 & 0 & 91 & 1 & 88 \\
\hline Liverant et al., 2016 & 0 & 39 & 0 & 48 \\
\hline Rodriguez-Sanchez et al., 2019 & 0 & 50 & 1 & 112 \\
\hline Schenck et al., 2017 & 0 & 73 & 0 & 62 \\
\hline Yamashina et al., 2019 & 0 & 108 & 0 & 102 \\
\hline Yen et al., 2019 & 0 & 68 & 0 & 50 \\
\hline Total $(95 \% \mathrm{Cl})$ & & 745 & & 769 \\
\hline Total events & 1 & & 2 & \\
\hline \multicolumn{5}{|c|}{$\begin{array}{l}\text { Heterogeneity: } \operatorname{Tau}^{2}=0.00 ; \mathrm{Chi}^{2}=0.96, \mathrm{df}=2(P=0.62) ; \mathrm{I}^{2}=0 \% \\
\text { Test for overall effect: } Z=0.12(P=0.90) \\
\text { b }\end{array}$} \\
\hline
\end{tabular}

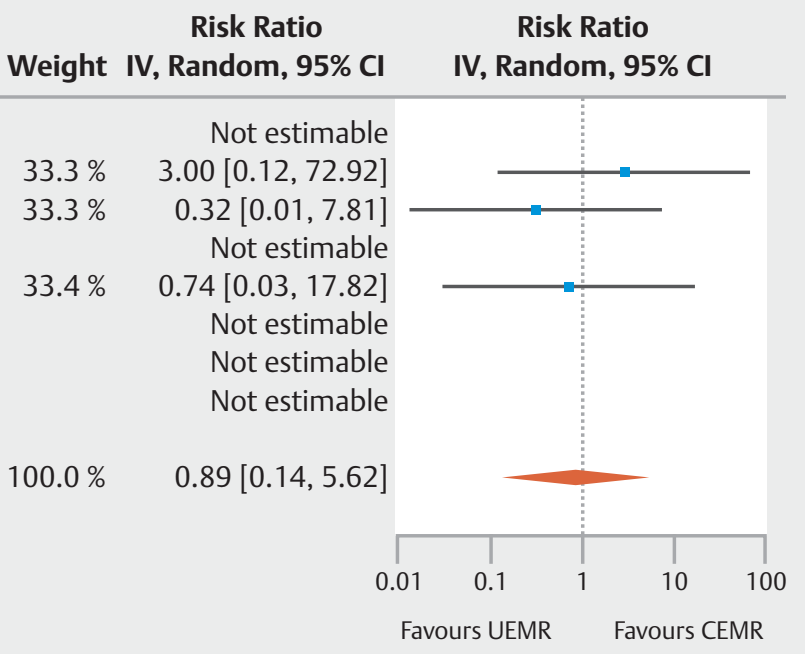

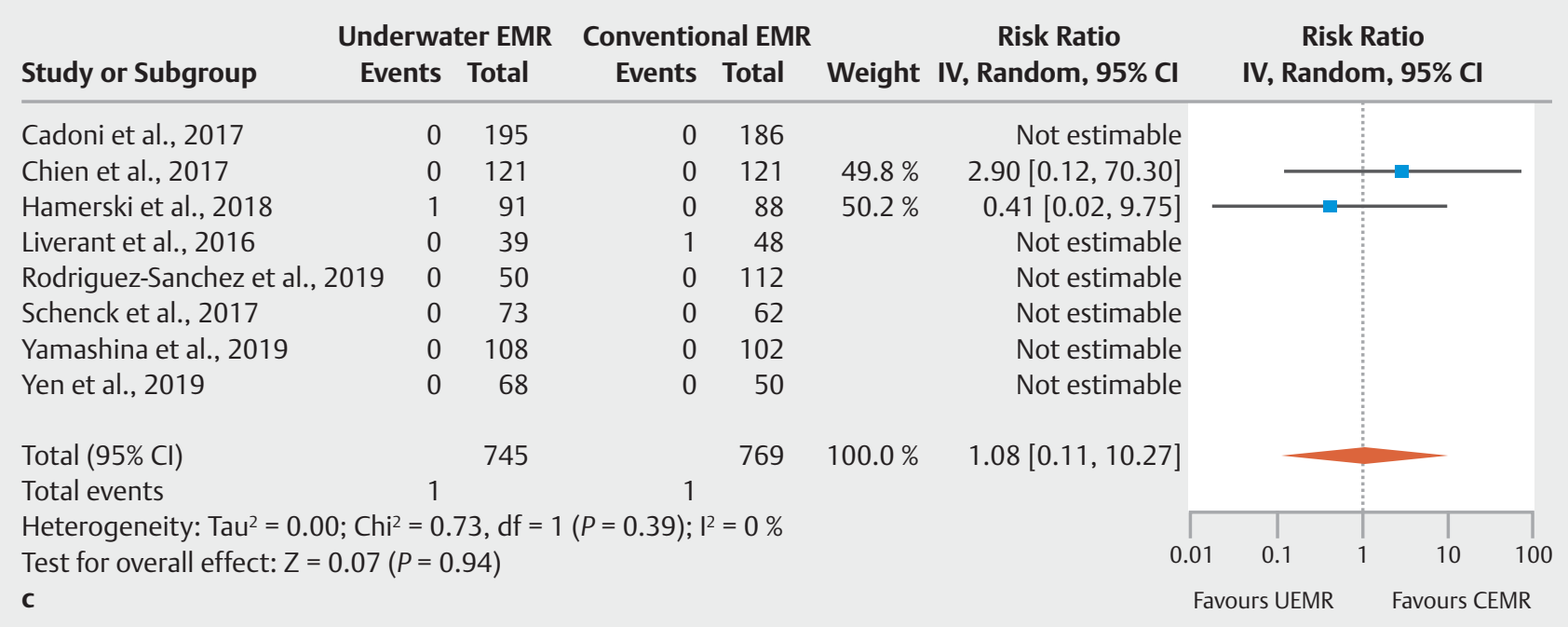

- Fig. 3 Forest plot showing relative risk of a delayed bleeding, b perforation, $\mathbf{c}$ and post-polypectomy syndrome comparing conventional and underwater endoscopic mucosal resection. 
- Table 3 Results of subgroup analysis of non-pedunclated polyps $\geq 10 \mathrm{~mm}$ and $\geq 20 \mathrm{~mm}$ comparing conventional endoscopic mucosal resection and underwater endoscopic mucosal resection.

\begin{tabular}{|l|l|l|}
\hline & Nonpedunclated polyps $\geq \mathbf{1 0} \mathbf{m m}^{1}$ & Nonpedunclated polyps $\geq \mathbf{2 0}$ mm ${ }^{\mathbf{1}}$ (OR) \\
\hline Incomplete resection (OR) & $0.26(0.03,2.54), P=0.11, I^{2}=55 \%, 3$ studies & $0.86(0.08,8.79), P=0.29, I^{2}=11 \%, 2$ studies \\
\hline Recurrence (OR) & $0.24(0.10,0.57), P=0.001, I^{2}=0,3$ studies & $0.14(0.02,0.72), P=0.01, I^{2}=11 \%, 2$ studies \\
\hline R0 resection (OR) & $1.62(0.86,3.04), P=0.15, I^{2}=52,2$ studies & 1 study \\
\hline Total complications (RR) & $0.70(0.48,1.03), P=0.07, I^{2}=12 \%, 7$ studies & $2.17(0.77,6.17), P=0.15, I^{2}=0 \%, 3$ studies \\
\hline Intra-procedural bleeding (RR) & $0.64(0.44,0.94), P=0.02, I^{2}=0 \%, 7$ studies & $0.85(0.15,4.73), P=0.85, I^{2}=74 \%, 4$ studies \\
\hline Delayed bleeding (RR) & $0.95(0.35,2.60), P=0.93, I^{2}=0 \%, 7$ studies & $1.77(0.23,13.34), P=0.84, I^{2}=0 \%, 3$ studies \\
\hline Perforation (RR) & $0.89(0.14,5.62), P=0.99, I^{2}=0 \%, 7$ studies & $0.89(0.09,8.39), P=0.92, I^{2}=0 \%, 3$ studies \\
\hline Post-polypectomy syndrome (RR) & $1.26(0.30,5.28), P=0.75, I^{2}=0 \%, 7$ studies & $0.92(0.13,6.41), P=0.93, I^{2}=0 \% 4$ studies \\
\hline En bloc resection (OR) & $1.44(0.74,2.78), P=0.28, I^{2}=84 \%, 7$ studies & $0.90(0.38,2.17), P=0.82, I^{2}=58 \%, 5$ studies \\
\hline${ }^{1}$ Results are odds ratio (OR) or relative risk (RR), $95 \%$ confidence interval, $I^{2}, P$ value and number of studies. & \\
\hline
\end{tabular}

In CEMR, increased colonic wall tension and gas insufflation also flattens the target lesion that complicates snare entrapment and can leave residual tissue. In addition, lesion flattening increases its overall surface area that might lead to more piecemeal resection. Underwater, the colon wall is involuted and has less area, thus, snare can potentially target and resect a larger lesion with UEMR. Needle tract seeding is also known risk in several endoscopic procedures and gastrointestinal malignancies [34,35]. Although rates may vary, there is a risk of submucosal microscopic seeding during submucosal injection in CEMR that can also contribute to higher rate of recurrence.

Submucosal injection in CEMR is performed to prevent deep thermal injury whereas UEMR utilizes natural collapsed state as water submersion decrease colonic wall tension and takes advantage of submucosal layer fat buoyancy which moves away from the muscularis propria, minimizing the risk of complications. In CEMR, needle puncture itself might precipitate bleeding, which possibly explain the significantly less risk of intraprocedural bleeding with UEMR [9]. This benefit of less intraprocedural bleeding was also noticed in non-pedunclated polyps $\geq 10 \mathrm{~mm}$ on subgroup analysis. CEMR may also lead to perforation and deep muscle injury if submucosal injection is misdirected. This can also be completely avoided with UEMR. Overall the rate of perforation was very low in both groups but UEMR does have these theoretical advantages. There was only one case of perforation after and 2 cases of perforation in CEMR group. On literature review, there was one additional case of perforation reported after UEMR whereas perforation rate of CEMR ranges from 0.8 to $1.5 \%[36,37]$. In our study, there was no significant difference in rates of delayed bleeding, perforation and PPS between both groups.

UEMR has also been shown reported to have less procedure time as compared to CEMR [26]. Although we were not able to directly compare the duration of procedure due to non-uniformity of data, but there was trend of less procedural time with UEMR. The UEMR duration ranged from 1.5 minutes to 13.3 minutes whereas CEMR duration ranged from 2.9 minutes to
26. 1 minutes. The less duration in UEMR is possibly explained by decreased rate of intra-procedural bleeding and absence of submucosal injection. CEMR is considered technically challenging on the right-sided lesions especially around appendiceal orifice due to thin wall and lack of muscularis mucosa. UEMR has also shown good results in resection of lesions at ileocecal valve and appendiceal orifice $[11,38]$. UEMR might also be valuable technique in tackling recurrent lesions. In one study comparing UEMR and CEMR, UEMR was superior in terms of higher rate of en bloc resection and fewer adenoma recurrences [39]. In fact, the rate of en bloc resection was almost $50 \%$ in that study, which is also comparable to $\operatorname{ESD}[39,40]$.

ESD is considered as treatment of choice for lesions greater than $20 \mathrm{~mm}$ with Paris Ilc or Ila + Ilc morphology or for any lesions greater than $3 \mathrm{~cm}$ per Japanese guidelines [41]. ESD is, however, limited by its high complications rate of $2 \%$ to $14 \%$ [36]. ESD is also technically challenging and time consuming, requires more training even by experienced endoscopists [ 2 , $42,43]$. UEMR can be easily learned and grasped by endoscopist's experienced in CEMR and can have quick uptake in the community practice.

Our review has many strengths including systematic literature search with well-defined inclusion criteria, careful exclusion of redundant studies, inclusion of good quality studies with detailed extraction of data and rigorous evaluation of study quality. The previous systematic review on UEMR did not provide data on non-pedunculated polyps and direct comparison between UEMR and CEMR [44]. There are limitations to this review, most of which are inherent to any meta-analysis. The studies were representative of centers in North and South America, Asia and Europe and not restricted to a geographic location. However, these studies were not entirely representative of the general population and community practice, with most studies being performed in tertiary-care referral centers. Our analysis had studies that were retrospective in nature contributing to selection bias and confounding bias. Although likely minimal, we could not account for intra-class correlation. The 
sample size of non-pedunculated polyps $>20 \mathrm{~mm}$ included in our study is small. Nevertheless, our study is the best available estimate in literature thus far, with respect to the clinical outcomes comparing UEMR and CEMR for resection of colorectal polyps.

\section{Conclusion}

In conclusion, our meta-analysis demonstrates that UEMR is associated with higher rate of complete resection and significantly fewer rate of recurrence. UEMR is also associated with significantly lower rates of adverse events and intra-procedural bleeding as compared to CEMR. These results were also significant for non-pedunculated polyps $>10 \mathrm{~mm}$. In expert hands UEMR could be preferred over CEMR.

\section{Acknowledgement}

The authors thank medical librarian Marian T. Simonson, MSLS, AHIP for assisting us in literature search.

\section{Competing interests}

The authors declare that they have no conflict of interest.

\section{References}

[1] Burgess NG, Bahin FF, Bourke M]. Colonic polypectomy (with videos). Gastrointest Endosc 2015; 81: 813-835

[2] Gaglia A, Sarkar S. Evaluation and long-term outcomes of the different modalities used in colonic endoscopic mucosal resection. Ann Gastroenterol 2017; 30: 145-151

[3] Ferlitsch M, Moss A, Hassan C et al. Colorectal polypectomy and endoscopic mucosal resection (EMR): European Society of Gastrointestinal Endoscopy (ESGE) Clinical Guideline. Endoscopy 2017; 49: 270-297

[4] Jayanna M, Burgess NG, Singh R et al. Cost analysis of endoscopic mucosal resection vs surgery for large laterally spreading colorectal lesions. Clin Gastroenterol Hepatol 2016; 14: 271-278 e271-272

[5] Knabe M, Pohl J, Gerges C et al. Standardized long-term follow-up after endoscopic resection of large, nonpedunculated colorectal lesions: a prospective two-center study. Am J Gastroenterol 2014; 109: 183-189

[6] Fukami N, Lee JH. Endoscopic treatment of large sessile and flat colorectal lesions. Curr Opin Gastroenterol 2006; 22: 54-59

[7] Moss A, Williams S], Hourigan LF et al. Long-term adenoma recurrence following wide-field endoscopic mucosal resection (WF-EMR) for advanced colonic mucosal neoplasia is infrequent: results and risk factors in 1000 cases from the Australian Colonic EMR (ACE) study. Gut 2015; 64: 57-65

[8] Binmoeller KF, Weilert F, Shah J et al. "Underwater” EMR without submucosal injection for large sessile colorectal polyps (with video). Gastrointest Endosc 2012; 75: 1086-1091

[9] Nett A, Binmoeller K. Underwater endoscopic mucosal resection. Gastrointest Endosc Clin N Am 2019; 29: 659-673

[10] Binmoeller KF. Underwater EMR without submucosal injection: Is less more? Gastrointest Endosc 2019; 89: 1117-1119
[11] Binmoeller KF, Hamerski CM, Shah JN et al. Underwater EMR of adenomas of the appendiceal orifice (with video). Gastrointest Endosc 2016; 83: 638-642

[12] Binmoeller KF, Hamerski CM, Shah JN et al. Attempted underwater en bloc resection for large $(2-4 \mathrm{~cm})$ colorectal laterally spreading tumors (with video). Gastrointest Endosc 2015; 81: 713-718

[13] Chien H-C, Uedo N, Hsieh P-H. Comparison of underwater and conventional endoscopic mucosal resection for removing sessile colorectal polyps: a propensity-score matched cohort study. Endosc Int Open 2019; 07: E1528-E1536

[14] Moher D, Liberati A, Tetzlaff J et al. Preferred reporting items for systematic reviews and meta-analyses: The prisma statement. Ann Int Med 2009; 151: 264-269

[15] Stroup DF, Berlin JA, Morton SC et al. Meta-analysis Of Observational Studies in Epidemiology (MOOSE) group. Meta-analysis of observational studies in epidemiology: a proposal for reporting. JAMA 2000; 283: 2008-2012

[16] Stang A. Critical evaluation of the Newcastle-Ottawa scale for the assessment of the quality of nonrandomized studies in meta-analyses. Europ J Epidemiol 2010; 25: 603-605

[17] DerSimonian R, Laird N. Meta-analysis in clinical trials. Controlled Clinical Trials 1986; 7: 177-188

[18] Higgins J, Thompson SG, Spiegelhalter DJ. A re-evaluation of randomeffects meta-analysis. J Royal Stati Society 2009; 172: 137-159

[19] Riley RD, Higgins JP, Deeks JJ. Interpretation of random effects metaanalyses. Br Med J 2011; 342: d549

[20] Mohan BP, Adler DG. Heterogeneity in systematic review and metaanalysis: how to read between the numbers. Gastrointest Endosc 2019; 89: 902-903

[21] Kanwal F, White D. Systematic reviews and meta-analyses. Clin Gastroenterol Hepatol 2012; 10: 1184-1186

[22] Higgins JP, Thompson SG, Deeks J] et al. Measuring inconsistency in meta-analyses. $\mathrm{Br}$ Med J 2003; 327: 557

[23] Guyatt GH, Oxman AD, Kunz R et al. GRADE guidelines: 7 Rating the quality of evidence and inconsistency. . J Clin Epidemiol 2011; 64: 1294-1302

[24] The Nordic Cochrane Centre TCC. Review Manager (RevMan) [Computer program]. Version 5.3. Copenhagen; 2014

[25] Cadoni S, Liggi M, Gallittu P et al. Underwater endoscopic colorectal polyp resection: Feasibility in everyday clinical practice. United European Gastroenterol J 2018; 6: 454-462

[26] Hamerski CM, Wang AY, Amato A et al. 121 Injection-Assisted versus underwater endoscopic mucosal resection without injection for the treatment of colorectal laterally spreading tumors: interim analysis of an international multicenter randomized controlled trial. Gastrointest Endosc 2018; 87: AB55-AB56

[27] Liverant ML, Yip B, Kwak N et al. Su1690 Underwater Endoscopic mucosal resection (EMR) shows a higher single session curative resection rate than conventional EMR technique: a single center experience. Gastrointest Endosc 2016; 83: AB397

[28] Rodriguez Sanchez J, Uchima Koecklin H, Gonzalez Lopez L et al. Short and long-term outcomes of underwater EMR compared to the traditional procedure in the real clinical practice. Rev Esp Enferm Dig 2019; 111: 543-549

[29] Schenck RJ, Jahann DA, Patrie JT et al. Underwater endoscopic mucosal resection is associated with fewer recurrences and earlier curative resections compared to conventional endoscopic mucosal resection for large colorectal polyps. Surg Endosc 2017; 31: 4174-4183

[30] Yamashina T, Uedo N, Akasaka T et al. Comparison of underwater vs conventional endoscopic mucosal resection of intermediate-size colorectal polyps. Gastroenterology 2019; 157: 451-461 e452

[31] Mouchli M, Walsh C, Reddy S et al. Sa1727 - Outcomes of gi polyps resected using underwater endoscopic mucosal resection (UEMR) 
Compared to conventional EMR (CEMR). Gastroenterology 2019; 156 : S379-S380

[32] Yen AW, Leung JW, Wilson MD et al. Underwater versus conventional endoscopic resection of nondiminutive nonpedunculated colorectal lesions: a prospective randomized controlled trial (with video). Gastrointest Endosc 2020; 91: 643-654 e642

[33] Oka S, Tanaka S, Saito Y et al. Local recurrence after endoscopic resection for large colorectal neoplasia: a multicenter prospective study in Japan. Am J Gastroenterol 2015; 110: 697-707

[34] Silva MA, Hegab B, Hyde C et al. Needle track seeding following biopsy of liver lesions in the diagnosis of hepatocellular cancer: a systematic review and meta-analysis. Gut 2008; 57: 1592-1596

[35] Yokoyama K, Ushio J, Numao $\mathrm{N}$ et al. Esophageal seeding after endoscopic ultrasound-guided fine-needle aspiration of a mediastinal tumor. Endosc Int Open 2017; 5: E913-E917

[36] Nakajima T, Saito Y, Tanaka S et al. Current status of endoscopic resection strategy for large, early colorectal neoplasia in Japan. Surg Endosc 2013; 27: 3262-3270

[37] Ponugoti PL, Rex DK. Perforation during underwater EMR. Gastrointest Endosc 2016; 84: 543-544
[38] Levy I, Hamerski CM, Nett AS et al. Su1618 underwater endoscopic mucosal resection (UEMR) of laterally spreading tumors involving the ileocecal valve. Gastrointestinal Endoscopy 2017; 85: AB366

[39] Kim HG, Thosani N, Banerjee S et al. Underwater endoscopic mucosal resection for recurrences after previous piecemeal resection of colorectal polyps (with video). Gastrointest Endosc 2014; 80: 1094-1102

[40] Sakamoto T, Matsuda T, Otake Y et al. Predictive factors of local recurrence after endoscopic piecemeal mucosal resection. J Gastroenterol 2012; 47: 635-640

[41] Tanaka S, Kashida H, Saito Y et al. JGES guidelines for colorectal endoscopic submucosal dissection/endoscopic mucosal resection. Dig Endosc 2015; 27: 417-434

[42] Saito Y, Uraoka T, Yamaguchi Y et al. A prospective, multicenter study of 1111 colorectal endoscopic submucosal dissections (with video). Gastrointest Endosc 2010; 72: 1217-1225

[43] Uraoka T, Parra-Blanco A, Yahagi N. Colorectal endoscopic submucosal dissection: is it suitable in western countries? J Gastroenterol Hepatol 2013; 28: 406-414

[44] Spadaccini M, Fuccio L, Lamonaca L et al. Underwater EMR for colorectal lesions: a systematic review with meta-analysis (with video). Gastrointest Endosc 2019; 89: 1109-1116 e1104 